IECEC 2002 Paper No. 20100

\title{
Microwave Power for Smart Membrane Actuators
}

\author{
Sang H. Choi \\ NASA Langley Research Center \\ Hampton, Virginia 23681-2199 \\ E-mail: S.h.chololarc.masa.gov \\ Kyo D. Song and Walter T. Golembiewski \\ Norfolk State University \\ Norfolk, Virginia 23504-3998 \\ Sang-Hyon Chu \\ ICASE, NASA Langley Research Center \\ Hampton, Virginia 23681-2199 \\ Glen C. King \\ NASA Langley Research Center \\ Hampton, Virginia 23681-2199
}

\begin{abstract}
The concept of microwave-driven smart membrane actuators is envisioned as the best option to alleviate the complexity associated with hard-wired control circuitry. A large, ultra-light space structure, such as solar sails and Gossamer spacecrafts, requires a distribution of power into individual membrane actuators to control them in an effective way. A patch rectenna array with a high voltage output was developed to drive smart membrane actuators. Networked patch rectenna array receives and converts microwave power into a DC power for an array of smart actuators. To use microwave power effectively, the concept of a power allocation and distribution (PAD) circuit is developed and tested for networking a rectenna/actuator patch array. For the future development, the PAD circuit could be imbedded into a single embodiment of rectenna and actuator array with the thin-film microcircuit embodiment. Preliminary design and fabrication of PAD circuitry that consists of a sixteen nodal elements were made for laboratory testing.
\end{abstract}

\section{INTRODUCTION}

The simplicity of a microwave-driven actuator system can reduce a total weight and fabrication cost of the system. A smart material actuator is utilized for a shape control of inflatable reflector antenna [1]. The shape control technique is extremely crucial to the success of NASA's future missions [2-5] including the Gossamer spacecrafts, Next Generation Space Telescope (NGST), space relay antennas, aircraft morphing, and insect-like flying objects. For a system with numerous nodal points that require power feed and control, the hard wiring may not be a suitable solution due to the weight increase attributed to the wired network, the complex gate switching of power and control networks needed, and the interdependency of power and control routines needed.

For the thin-membrane structure applications, several types of rectennas were reviewed in terms of power conversion efficiency, flexibility, and fabrication requirements. Most smart materials, such as piezoelectric or electro-active, require from 200 volts to 300 volts to activate appreciable shape changes. In our early rectenna development, a $3 x$ 3 array of patch rectennas used for a multilayered piezoelectric actuator had a capacity to generate 70 volts output $[4,5]$.

Since the rectenna was first introduced by W. C. Brown in the 1960's [6], it has been used for various applications such as a microwave-powered helicopter and model airplanes [7], and proposed for the Solar Power Satellite (SPS) [8] that converts solar energy to RF and beams down to large arrays of $5-\mathrm{GHz}$ rectennas on Earth, the 4.5-meter wingspan airplane that was powered only by 
microwave energy [9], and a microwave-powered balloon with an electronically steerable phased array [10]. Recently, a lightweight patch rectenna was developed at NASA's Jet Propulsion Laboratory [11] for smart actuator applications. Its structure is composed of a square planar array of identical unit cells. Each cell contains a receiving antenna, filter and rectifier circuitry in its planar structure. Microwave coupling between each copper antenna patch and its underlying filter and rectifier circuit occurs in two orthogonal slots in the copper ground plane.

In practical applications, the power received by a single element of patch rectenna array may not be sufficient for driving even an actuator element because of the low incident microwave power on a patch rectenna. The power output from patch rectennas is determined by the power flux density, frequency, and incident angle of incident microwave and the rectifying circuit performance. The breakdown voltage of Schottky barrier diodes used in each individual rectenna is also a limiting factor. Accordingly, devices operating at a low power level must intelligently manage the power to meet user device requirements. The issues related to low power density of microwave and power demand can be alleviated by employing the concept of power allocation and distribution (PAD).

\section{Experimental Results}

Microwave Power Density Distribution - An experimental was setup to actuate THUNDER (Thin Layer Composite Unimorph Ferroelectric Driver and Sensor) actuators using a microwave power as shown in Fig.1. A combination of signal generator and amplifier provided $20 \mathrm{~W}$ microwave power to a Narda horn antenna at $8.5 \mathrm{GHz}$. The $20 \mathrm{~W}$ microwave power irradiated a JPL's $6 \times 6$ rectenna array. The typical spatial distribution of the microwave power density on the rectenna is measured at a distance from the horn inside of the anechoic chamber as shown Fig. 2. As a result, the average power density of a $20 \mathrm{~W}$ microwave at 8.5 $\mathrm{GHz}$ is approximately $5 \mathrm{~mW} / \mathrm{cm}^{2}$. The foot-print area of microwave at 1 meter away is approximately $2500 \mathrm{~cm}^{2}$ where $60 \%$ of the microwave power beam delivery was measured. A horn antenna and a $6 \times 6$ rectenna array with a fixture were set up inside the anechoic chamber as shown in Fig. 3 (a) and (b).

Output Power of $6 \times 6$ Rectenna - A $6 \times 6$ patch rectenna was used to achieve the higher voltage output requirement. The voltage output of patch rectenna is determined by the number of inbedded Schottky barrier diode on a rectifying circuit. Each patch has two of Schottky barrier diodes embedded in a serial mode with a hold-off voltage of 15 volts. Thus, the $6 \times 6$ patch rectenna is, theoretically, able to generate the output voltage up to 540 volts. The actual voltage output is lower than the theoretical value and determined by the electrical properties of substrate materials on which a rectifying circuit is built. The performance of the $6 \times 6$ patch rectenna is shown in Figs $4-6$. The output power $\left(\sim V^{2} / R_{L}\right)$ from the $6 \times 6$ patch rectenna module with constant load resistance $\left(R_{L}\right)$ were measured at a far-field along the distance from the feed horn and plotted in terms of voltage output versus distance as shown in Fig. 4. The input power dependent voltage outputs were also measured at the distance of $100 \mathrm{~cm}$ away from a feed horn as shown in Fig. 5. In fig. 6, the output voltage versus microwave input power are measured and plotted. The peak voltage output was 230 volts at $8 \mathrm{GHz}$ with 20 watts of microwave beam power. The peak voltage output is within the voltage requirement for most piezoelectric actuators.

Actuation of THUNDER Actuators - A THUNDER actuator is tested with a $6 \times 6$ patch rectenna array is shown in Fig. 1. In this experiment, the $20 \mathrm{~W}$ microwave power was converted into a measured $230 \mathrm{~V}$ DC by a digital multimeter. The estimated current being produced from the $6 \times 6$ rectenna array was $0.38 \mathrm{~mA}$. This was computed using various measured resistor values and the measured voltage across each resistor.

THUNDER is a ferroelectric device made of multiple layers of materials, typically stainless steel, aluminum and PZT (Lead Zirconate Titanate) piezoceramic. These layers of materials are sandwiched together with an adhesive bond. A piezoceramic material is composed of randomly oriented crystals or grains. By a strong DC electric field, the dipoles are aligned in the direction of the electric field. The result of this polarization appears as a change in the geometric dimension. Through the piezoelectric effect, THUNDER has the capability to expand or contract, based on the polarity of the voltage applied. When the applied voltage is positive, THUNDER will flatten, and if the applied voltage were negative, the THUNDER arches. The THUNDER used for the test rated a 31 $\mathrm{Hz}$ resonant frequency, a 595 volts driving voltage, and a $7.62 \mathrm{~mm}$ maximum displacement. Fig. 7 shows the displacement levels of THUNDER actuator as a function of the $6 \times 6$ patch rectenna array output voltage. A higher voltage output than 230 volts is expected by increasing the beam power. The test of the $6 \times 6$ patch rectenna was limited by the capacity of the $20 \mathrm{~W}$ tunneling wave tube amplifier (TWTA) used. 
Currently, a 200 W TWTA is under installation and expected for higher voltage output from the $6 \times 6$ patch rectenna as predicted in Fig. 8. High voltage output is, generally, determined by the number density and breakdown voltage of Schottky barrier diodes in a rectenna array, the beam power, the frequency, and the distance, respectively. The number density of Schottky diodes is determined by how patch rectennas are densely packed in an array at a given frequency.

A 4 × 4 PAD Circuit Fabrication - The PAD circuitry utilizes most of microwave power at the rectenna's maximum efficiency. It tailors the power mode and boosts either output voltage or output current for actuator array. A $4 \times 4$ grid of MOSFET's was constructed by connecting all the number two gates (G2) of four MOSFET's together in the first row. It takes four row wires and four column wires to control sixteen MOSFET's and the corresponding actuators. In this design, a digital to analog converter (DAC) board in a standard computer was used to turn on rows and columns wires in a roundrobin fashion. A separate analog channel was used for each row or column. The required number of row or column wires is given by the square root of the number of actuators.

The power distribution to a designated group of actuators can be controlled by the external command signals. A network circuitry that interconnects control logic of all participating rectennas allocates power that is received by individual rectenna's of the array to a group of actuators from one location to another location. The networked allocation of power is also a function of the process of the power amplification corresponding to the power needs for a group of actuators. Within the group of actuators, the power allocated to the group is distributed to each actuator of the group according to power need. The actual 4 x 4 PAD system developed is shown as in Fig. 9.

Visual Basic Control Program - A Visual Basic program (Version 6.0) is used to control the digital to analog output board. A typical display for $4 \times 4$ actuator for control panel (a) and output (b) is shown in Fig. 10. In Fig. 10 (a), red boxes indicate actuators that power is on, while the green boxes show there is no power on the actuators. Similarly, the red circles show level of the output power in Fig. 10 (b). The bigger circles showed higher output power on each actuator.

\section{CONCLUSION}

The experimental results indicate that the rectenna array generates sufficient output power to drive presently available actuator like THUNDER. The wireless power transmission by microwave eliminates hard-wires networking actuator elements on the adaptive surfaces of a large space deployable reflectors or inflatable antennas. Hence, it could dramatically reduce the weight and cost of distributed shape-control. The conceptual design of PAD presented is a reasonable solution to the complexity of wiring many actuators. For higher power applications, high voltage hold-off Schottky barrier diodes and MOSFETs are essential for driving actuators with a large displacement requirement, such as aircraft morphing actuators.

\section{ACKNOWLEDGMENTS}

This work was performed partially under the NASA grant (NCC1-280) to Norfolk State University.

\section{REFERENCES}

1. T. K. Wu, "Multiband FSS," in T. K. Wu (Ed.), Frequency Selective and Grid Array, John and Wiley \& Sons, New York, 1995.

2. W. Schneider, J. Moore, T. Blankney, D. Smith, and J. Vacchione, "An Ultra-Lightweight High Gain Spacecraft Antenna," IEEE Antennas Propagat. Int. Symp., Seattle, WA, June, pp. 886-889, 1994.

3. T. H. Lee, R. C. Rudduck, T. K. Wu, and C. Chandler, "Structure Scattering Analysis for SeaWinds Scatterometer Reflector Antenna Using Extended Aperture Integration and GTD," IEEE Antennas propagat. Int. Symp., pp. 890-893, Seattle, WA, June, 1994.

4. Sang H. Choi, Lake, M., and Moore, C., "Microwavedriven Smart Material Actuators." Patent Pending, NASA Case No. LAR 15754-1, Feb 24, 1998

5. Sang H. Choi, S. Chu, M. Kwak and A. D. Cutler, "A Study on a Microwave-driven Smart Material Actuator," SPIE Symposium on Smart Structures and Materials, 1999

6. W. C. Brown, et al., U.S. Patent 3434 678, Mar. 25 , 1969.

7. W. C. Brown, "Experiments involving a microwave beam to power and position a helicopter," IEEE Trans. Aerosp. Electron. Syst., Vol. AES-5, No. 5, pp. 692-702, 1969.

8. W. C. Brown, "Solar Power Satellite Program Rev. DOE/NASA Satellite Power System Concept Develop. Evalation Program," Fianl Proc. Conf. $800491,1980$.

9. J. Schlesak, A. Alden and T. Ohno, "A microwave powered high altitude platform," IEEE MTT-S Int Microwave Symp. Dig., pp. 283-286 1988.

10. W. C. Brown, "Design study for a ground microwave power transmission system for use with a highaltitude powered platform," NASA Final Contractor Report 168344, Raytheon Rpt. PT-6052, 1983.

11. NASA JPL, "Patch Rectenna for Converting Microwave to DC Power," NASA Tech Briefs, Vol. 21 January, p. 40, 1997. 


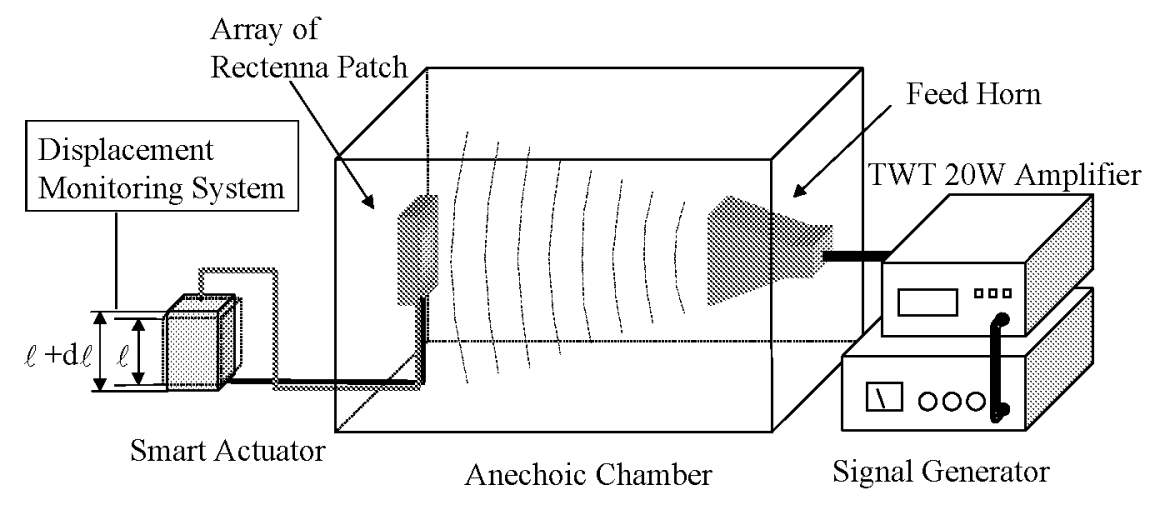

Fig. 1. Experimental setup for microwave-driven Thunder Actuator

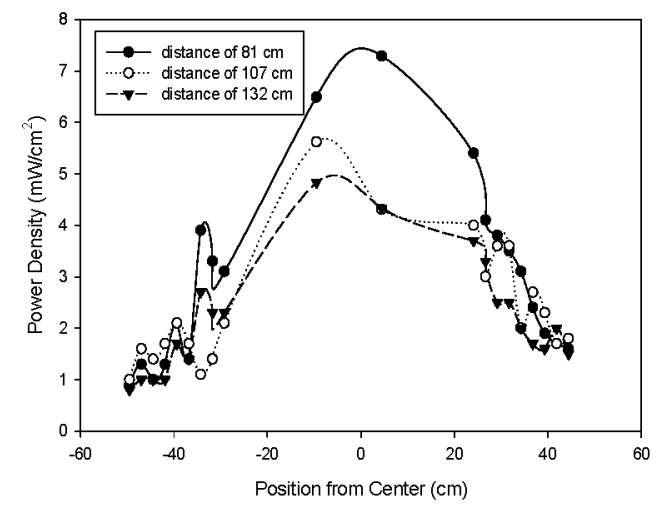

Fig. 2. Microwave Power Density Distribution along Distances

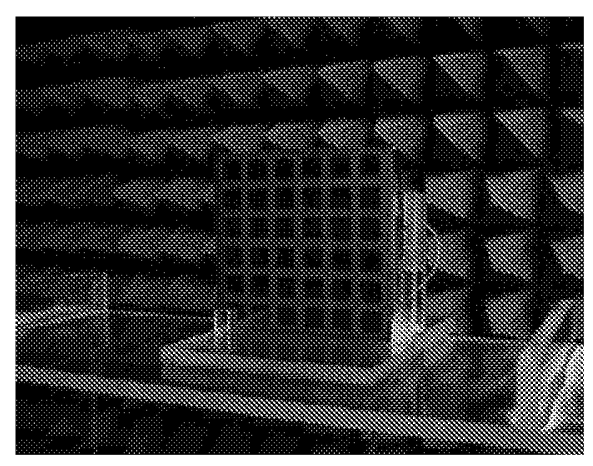

Fig. 3b. 6 × 6 rectenna set up ( showed as front view) with its mounting

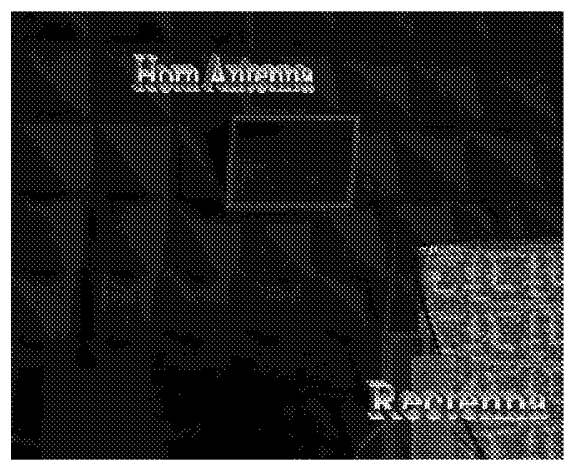

Fig. 3a. $6 \times 6$ rectenna (showed as rear view) set up in anechoric chamber with a horn antenna

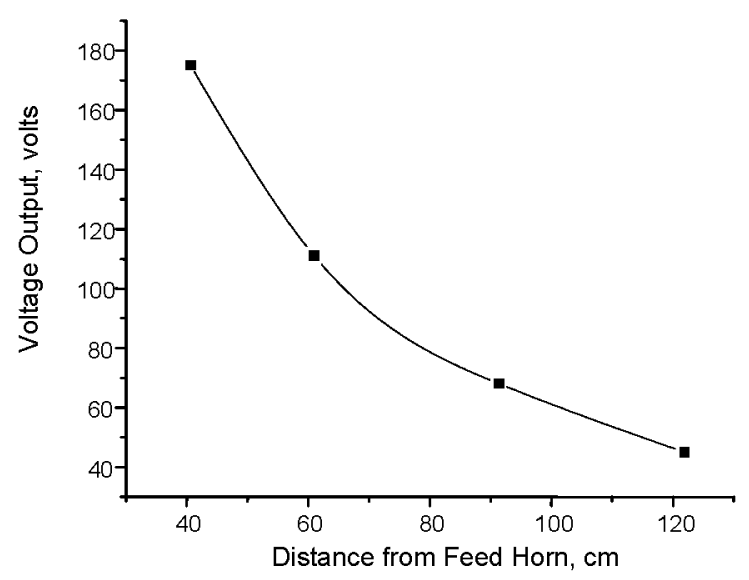

Fig. 4. Voltage outputs from a $6 \times 6$ patch rectenna array with distance from feed horn 


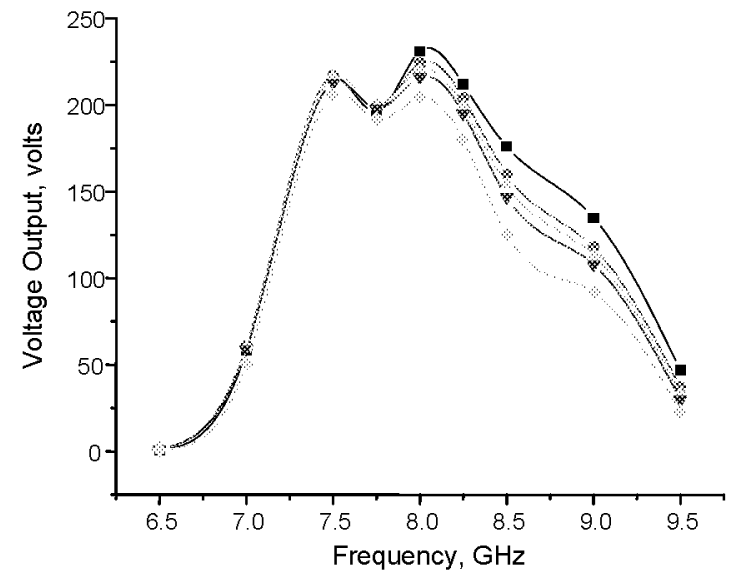

Fig. 5. Frequency dependent voltage output from a $6 \times 6$ patch rectenna array

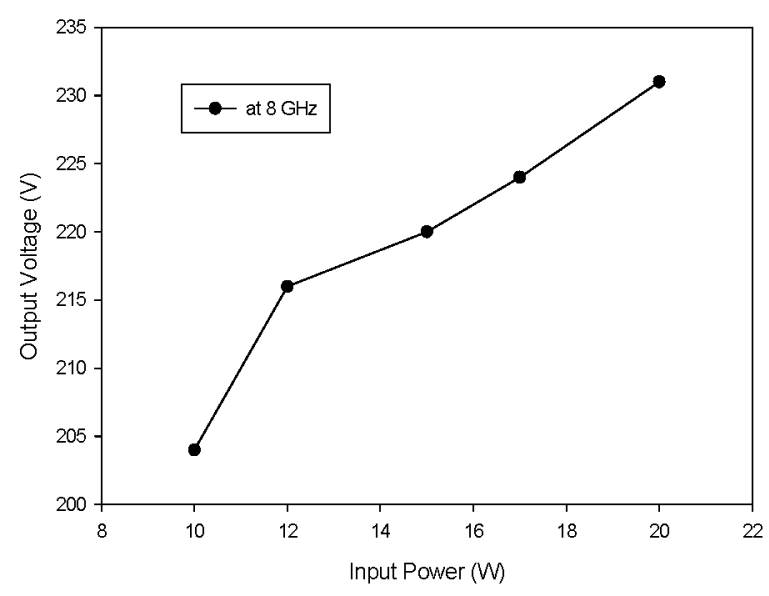

Fig. 6. Input power dependent DC output voltage from a $6 \times 6$ patch rectenna

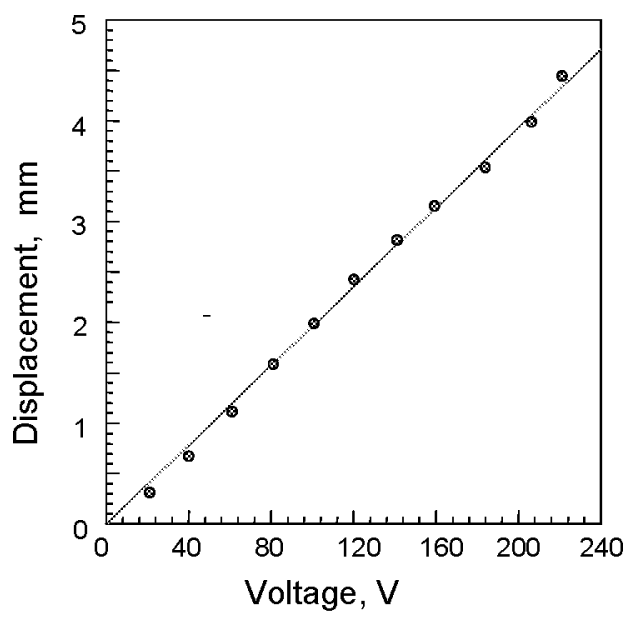

Fig. 7. Displacement of THUNDER actuator

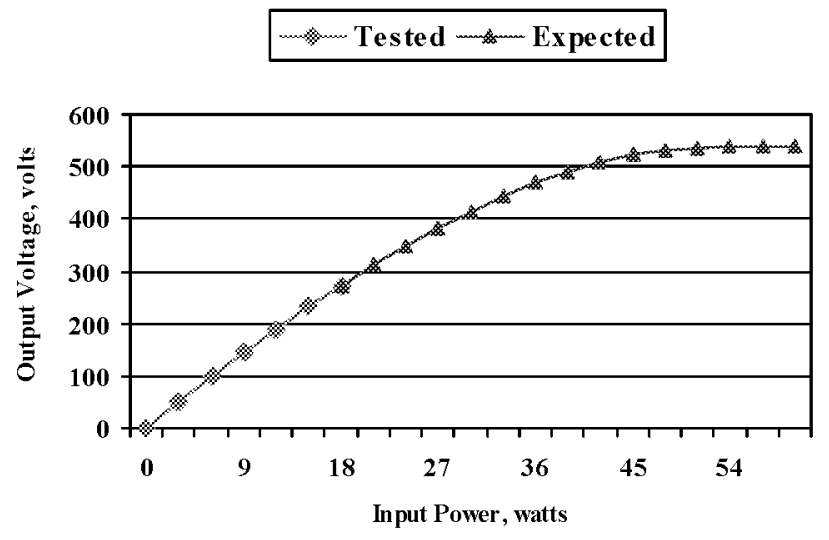

Fig. 8. Output Voltage from a $6 \times 6$ rectenna patch in terms of input power of a microwave 


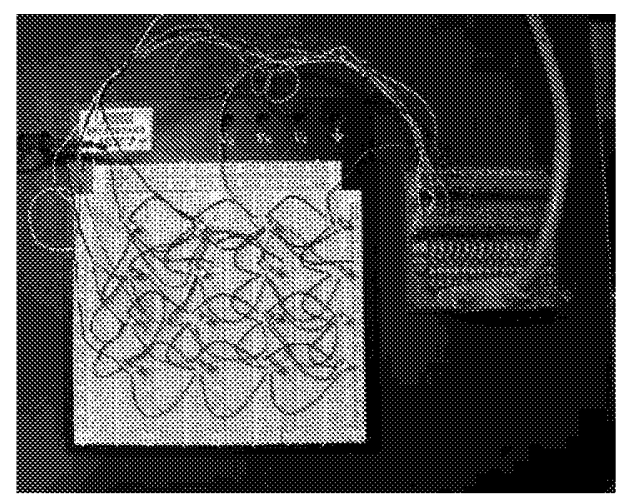

Fig. 9. A picture of a $4 \times 4$ PAD system

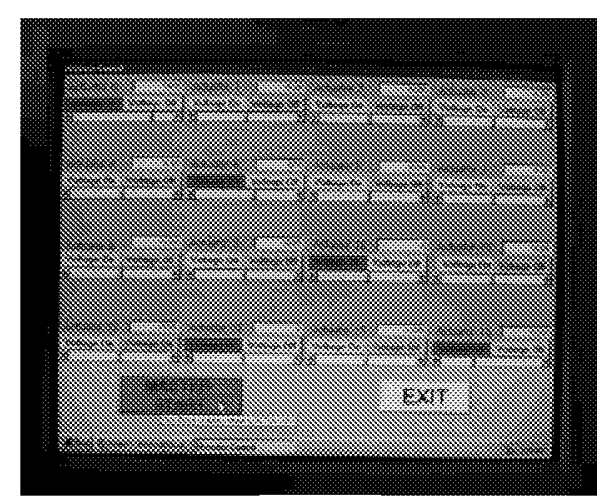

(a)

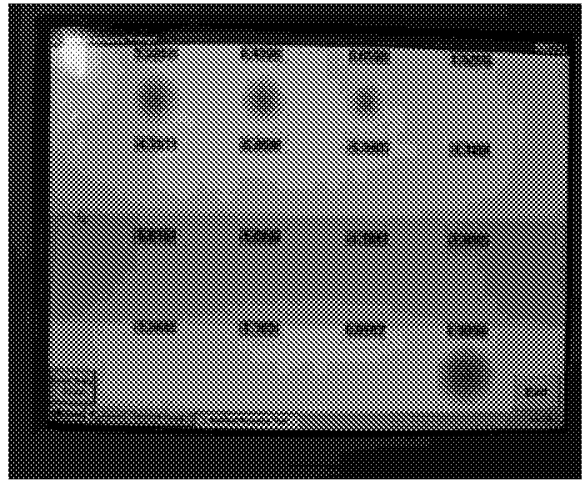

(b)

Fig. 10. Computer display for $4 \times 4$ actuator control. (a) Control display panel (b) Display output 